\title{
Additional Utility of Pharmacogenomics (PGx) Panel Testing in a CYP2D6 Normal Metabolizer with a History of Breast Cancer
}

Michael J. Schuh, PharmD, MBA, FAPhA

Family Medicine and Palliative Medicine, Mayo Clinic Florida

\begin{abstract}
Objective: To demonstrate the utility of pharmacogenomic (PGX) panel testing use versus single gene testing for a single indication. Panel testing may not only help further refine clinical decision making for the primary medical indication, it may uncover with one diagnostic test multiple PGX abnormalities, altering current and future therapy for other conditions.

Summary: Breast cancer patient presented to the pharmacist PGX service to discuss results and to help determine best guidance for post-surgical pain treatment. From the panel testing it was incidentally found the patient may be at higher clot risk from standard cancer prophylactic, hormone therapy as well as possible future cardiac therapy.

Conclusion: PGx panel testing may not only uncover potential medication related problems (MRPs) with the primary medical indication being tested, it may also refine therapy for other medical problems, resulting in avoidance of future MRPs and the health care costs associated with them.
\end{abstract}

Key words: Pharmacogenomics (PGx), Medication therapy management (MTM), Adverse drug reactions (ADRs), Factor V Leiden thrombophilia, Tamoxifen, CYP2D6, Comprehensive medication review (CMR), CYP2C19

\section{Introduction}

As pharmacogenomics (PGx) testing has become more extensive, less expensive and more available, there has been a trend in using panels for testing. ${ }^{1-2}$ Some concerns with panel testing include the varying degrees of evidence available for the genes tested for on a given panel and the higher cost associated with a panel testing, especially if genes are not related to a patient's specific condition being treated..$^{3-5}$ Some literature indicates PGx panel testing may be a cost effective strategy for patient treatment. ${ }^{2,6-7}$ In this example of PGx panel testing, significant, actionable results were found incident to the primary objective of the pharmacist PGx consult.

Here we present a patient with breast cancer that underwent previous PGx testing while participating in a PGx study for better pain management therapy after breast cancer surgery. Though the primary objective of the PGx lab panel testing was pain management, the panel revealed indicators of possible increased clot risk through Factor $V$ Leiden thrombophilia (F5) and CYP2C19 intermediate metabolizer status. ${ }^{3,8}$ These results may impact breast cancer prophylaxis (PPx) therapy after breast surgery. ${ }^{8}$ Secondarily, as a CYP2C19 intermediate metabolizer, inability to convert clopidogrel to the active form

Corresponding author: Michael J. Schuh, PharmD, MBA, FAPhA Assistant Professor of Pharmacy

Family Medicine and Palliative Medicine

Mayo Clinic Florida

4500 San Pablo Rd., Jacksonville, FL 32224

Work: 904-953-2673; Fax: 904-953-2274

E-mail: Schuh.michael@mayo.edu may increase clotting, even with treatment after a cardiac event or stroke. ${ }^{3}$ Standard breast cancer PPx therapy after surgery is tamoxifen or an aromatase inhibitor (AI) such as letrozole, anastrozole or exemestane. Though this patient had normal CYP2D6 activity, tamoxifen is thought to have a higher risk of thrombosis than Als and patients with F5 and a history of clots may be at even higher risk. ${ }^{9-10}$ The impact of the nonconversion of clopidogrel to the active form as a CYP2C19 intermediate metabolizer is more relevant since she is at a higher risk for clot formation due to her F5 status

\section{Setting}

The practice setting is an interdisciplinary, pharmacist office practice located in a multi-specialty, tertiary care clinic in Florida. The clinic offers pharmacist-provided medication therapy management (MTM), including more advanced PGx services to all patients in the clinic via referral from physicians and other providers with ordering privileges. More advanced in this case is defined as PGx results applied to comprehensive medication reviews (CMRs), including herbal supplements and cannabis products. The pharmacist-run PGx service sees patients from more than 20 different departments and is considered a specialty service, similar to other specialty services within the clinic. The PGx pharmacist patient visits occur within the provider offices to review PGx lab results. (Table I)

\section{Case Report}

Patient is a 52-year-old white female with newly discovered breast cancer. The breast tumor was discovered incidentally. Surgery was performed to remove the tumor and patient was scheduled to see the pharmacist for a PGx consult the next day. Previously, her surgeon enrolled her in a PGx study and ordered 
PGx testing to assist in pain management post-surgery. (Table I) This surgeon is an institution PGx champion and has discovered panels are useful in pain management post-op and in the discovery of unrelated, actionable medication related problems (MRPs). The ordering of the panel testing begins with an order being placed in the electronic medical record (EMR). The patient then either goes to the onsite lab to provide a cheek swab DNA sample or a lab kit is sent to the patient's home for them to provide the cheek swab sample there to mail back to the lab. If the sample is obtained at home, a return envelope is provided the patient in the lab kit so they can return the sample to the lab. Onsite lab results are available in as little as eight days, kits sent to the patient's home usually takes two to three weeks. This patient was enrolled in a pain study and the incidental findings of this case occurred when the patient was interviewed as a study patient.

A PGx panel of 27 genes was utilized and findings reviewed with the patient by the PGx pharmacist. A sample of what such a panel would look like is provided. (Figure I) Though there were no significant gene-drug interactions associated with pain management, significant, incidental findings were discovered relating to other current and potentially future therapies. Notably after PGx panel testing, the patient was found to be at increased risk of thrombosis associated with Factor $V$ Leiden (F5). Intermediate CYP2C19 status indicated poor conversion of clopidogrel to the active form to prevent clotting with clopidogrel treatment. Upon interacting with the patient it was also discovered she had clot formation after a previous surgical procedure.

PGx panel testing indicated she is an extensive (normal) metabolizer of CYP2D6. CYP2D6 is the primary gene that produces the enzyme converting tamoxifen from the inactive form to the active form endoxifen. ${ }^{3}$ This active form competes with estrogen for receptor sites in certain breast cancers to block the proliferative effect on cancer cell growth. ${ }^{11}$ Tamoxifen is standard therapy in patients who have had breast cancer surgery and/or radiation treatment. It is typically taken for 5 years after initial treatment for cancer PPx to prevent the cancer from recurring. ${ }^{11}$ CYP2D6 normal metabolizers metabolize tamoxifen normally so there is usually no reason to not use tamoxifen cancer recurrence PPx. ${ }^{3}$

\section{Discussion}

According to CPIC guidelines, an extensive CYP2D6 metabolizer should use tamoxifen for PPx therapy after breast cancer surgery. However, the incidental findings from the PGx panel for this patient and past medical history revealed an F5 patient with a history of clotting. With tamoxifen having a higher risk of clots than Als, tamoxifen use was reconsidered. As recommended by the PGx pharmacist, the patient was started on the aromatase inhibitor anastrozole for breast cancer PPx. In this patient, PGx panel testing revealed three important therapeutic issues that may not have been discovered otherwise. The first was a possible explanation of previous clotting after a past surgical procedure. Second, an F5 positive finding on her PGx results guided therapy away from the higher clot risk tamoxifen to the lower clot risk aromatase inhibitor, anastrazole. ${ }^{8}$ And finally, intermediate CYP2C19 status disqualifies future clopidogrel use if there is a future indication and indicates the use of prasugrel or ticagrelor. ${ }^{3}$ It is imperative that if the patient would require an antiplatelet therapy in the future, clopidogrel would not be used due to the inability for the patient to activate the drug. This is especially important given the patient's increased risk for thrombosis owing to their F5 status. F5 deficiency has been shown to increase myocardial infarction (MI) risk and standard therapy after $\mathrm{MI}$ is clopidogrel. ${ }^{11-12}$

Using a multi-gene panel versus a single gene testing may also limit health care costs. Most PGx testing is permanent so results may be used for the patient's lifetime without retesting. Panel testing may help predict future MRPs and provide an opportunity to avoid them, thus saving health care dollars resulting from decreased emergency room visits and hospitalizations. Incidental costs saved would be the cost of PGx retesting if a single gene was tested versus a panel of genes.

\section{Case Summary}

Patient was enrolled for a PGx panel study for guided pain management post-surgery for a breast cancer mass. The PGx panel was intended for pain management after surgery. Incidental findings from the PGx panel results revealed that the patient was at greater clot risk due to F5 status with normal post-surgery breast PPx therapy tamoxifen. As such, the PPx therapy was changed to the alternative aromatase inhibitor, anastrozole. The patient's PGx panel also confirmed the need to avoid future therapy with clopidogrel if this agent is ever warranted because of dual factors of F5 with clotting and CYP2C19 intermediate metabolizer status.

\section{Conclusion}

PGx panel testing is controversial with regard to utility and third party payment because of varying levels of evidence and nonspecificity of the panel to targeted therapy. Patient's original indication for testing was for pain management. However, multiple other MRPs were discovered with PGx panel testing when the primary objective of the PGx lab test results was unremarkable. With only single gene, CYP2D6, PGx lab testing alone, this patient's post-cancer treatment PPx therapy may have been straightforward. However, the case demonstrates that even in a seemingly straightforward case of established treatment, mitigating factors from PGx panel testing may be incidentally discovered that may further refine therapy to attain better therapeutic outcomes or to avoid possible, future adverse drug reactions. PGx panel testing can also save health care dollars by avoiding future emergency room visits, hospitalizations or retesting genes in the future for other indications. 


\section{Conflict of Interest: None}

\section{References}

1. Stanek EJ, Sanders CL, Johansen Taber KA, Khalid M, Patel A, Verbrugge RR, Agatep BC, Aubert RE, Epstein $\mathrm{RS}$, Frueh FW. Adoption of pharmacogenomic testing by U.S. physicians: results of a nationwide survey. Clin Pharmacol Ther. 2012 Jan;91(3):450-458.

2. Reiling MV, Evans WE. Pharmacogenomics in the clinic. Nature. 2015 Oct 14;526:343-350.

3. Whirl-Carrillo M, McDonagh EM, Hebert JM, Gong L, Sangkuhl K, Thorn CF, Altman RB, Klein TE.

"Pharmacogenomics knowledge for personalized medicine" Clinical Pharmacology \& Therapeutics (2012);92(4):414-17.

4. Lu CY, Loomer S, Ceccarelli R, Mazor KM, Sabin J, Clayton EW, Ginsburg, GS, Wu AC. Insurance coverage policies for pharmacogenomic and multigene testing for cancer. J Pers Med. 2018 Jun:8(2):19.

5. CMS.gov. Centers for Medicare and Medicaid Services. Decision Memo for Pharmacogenomic testing for warfarin response (CAG-00400N). Retrieved March 4, 2019, from https://www.cms.gov/medicare-coveragedatabase/details/nca-decisionmemo.aspx ?NCAld $=224 \&$ ver $=15 \&$ NcaName $=$ Pharmac ogenomic+Testing+for+Warfarin+Response \&bc=ACA AAAAAIAAA\&.

6. Verbelen M, Weale ME, Lewis CM. Cost-effectiveness of pharmacogenetic-guided treatment: are we there yet? Pharmacogenomics J. 2017 Oct;17(5):395-402.
7. Haga S, Moaddeb J. Comparison of delivery strategies for pharmacogenetic testing services. Pharmacogenet Genomics. 2014 Mar;24(3):139-145.

8. Cuzick J. Tamoxifen and factor V leiden mutation. J Natl Cancer Inst. 2010 Jul:102(13):918-919

9. Bundreed NJ. The effects of aromatase inhibitors on lipids and thrombosis. Br J Cancer. 2005 Aug; 93:S2327.

10. Weitz IC, Israel VK, Liebman HA. Tamoxifenassociated venous thrombosis and activated protein $C$ resistance due to factor $\mathrm{V}$ leiden. Cancer. 2000 Sep;79(10):2024-2027.

11. Spoon MB, Lippman SM. Agents for chemoprevention and their mechanism of action. Holland-Frei Cancer Medicine. $6^{\text {th }}$ Edition. Retrieved March 4, 2019, from https://www.ncbi.nlm.nih.gov/books/NBK12522/.

12. Dowaidar $M$, Settin A. Risk of myocardial infarction related to factor $\mathrm{V}$ leiden mutation: a meta-analysis. Genet Test Mol Biomarkers. 2010 Aug;14(4):493-8. doi: $10.1089 /$ gtmb.2010.0017.

13. Levine GN, Bates ER, Bittl JA, et al. ACC/AHA Guideline update on duration of dual antiplatelet therapy in CAD patients. J AM Coll Cardiol. 2016 Sep;68(10). DOI: 10.1016/j.jacc.2016.03.513. Retrieved April 26, 2019, from http://www.onlinejacc.org/content/68/10/1082?_ga $=2.83536982$.1583336994.15562976881211587098.1548419842.

14. OneOme. Retrieved June 12, 2019, from www.OneOme.com. 
Table I

Relevant Patient PGx Results

\begin{tabular}{|l|l|l|l|l|}
\hline \multicolumn{1}{|c|}{ Gene } & \multicolumn{1}{|c|}{ Function } & \multicolumn{1}{c|}{ Genotype } & \multicolumn{1}{c|}{ Phenotype } & \multicolumn{1}{c|}{ Interpretation } \\
\hline CYP2C19 & PK drug metabolism & $* 1 /{ }^{*} 2$ & Intermediate & $\begin{array}{l}\text { Decreased activity. Drugs converted to active } \\
\text { metabolite(s) may have reduced efficacy. Active } \\
\text { drugs converted to inactive metabolite(s) may } \\
\text { cause side effects or toxicity }\end{array}$ \\
\hline CYP2D6 & PK drug metabolism & $* 1 /{ }^{*} 2 \mathrm{~A}$ & Extensive (Normal) & $\begin{array}{l}\text { Normal level of activity. Drugs metabolized at a } \\
\text { normal rate }\end{array}$ \\
\hline F5 & PD (Clotting) & rs6025 GA & Increased risk & $\begin{array}{l}\text { Increased risk of thrombosis associated with } \\
\text { Factor V Leiden thrombophilia versus normal risk }\end{array}$ \\
\hline OPRM1 & PD activity (Pain) & rs1799971 AA & $\begin{array}{l}\text { Minimal gene-drug } \\
\text { interaction }\end{array}$ & $\begin{array}{l}\text { OPRM1 Asn/Asn (AA) genotype associated with } \\
\text { normal to increased sensitivity to the analgesic } \\
\text { effects of alfentanil, codeine, fentanyl, } \\
\text { morphine, and tramadol compared to patients } \\
\text { with the OPRM1 AG or GG genotypes at } \\
\text { rs1799971. }\end{array}$ \\
\hline COMT & PD activity (Pain) & rs4680 GG & $\begin{array}{l}\text { Minimal gene-drug } \\
\text { interaction }\end{array}$ & $\begin{array}{l}\text { COMT activity with GG genotype is predicted to } \\
\text { be normal }\end{array}$ \\
\hline
\end{tabular}

Note:

Nomenclature for genotypes can be the * alleles from each parent separated by a slash (i.e. ${ }^{*} 1 /{ }^{*} 2$ ) or single nucleotide polymorphism (SNP) at a marked gene location (i.e. SNP = GA, location $=$ rs6025)

Source of genotype, interpretation: www.OneOme.com ${ }^{14}$

$\mathrm{PK}=$ Pharmacokinetic, $\mathrm{PD}=$ Pharmacodynamic 
Case Study

PHARMACY PRACTICE

Figure I

PGP Sample Report

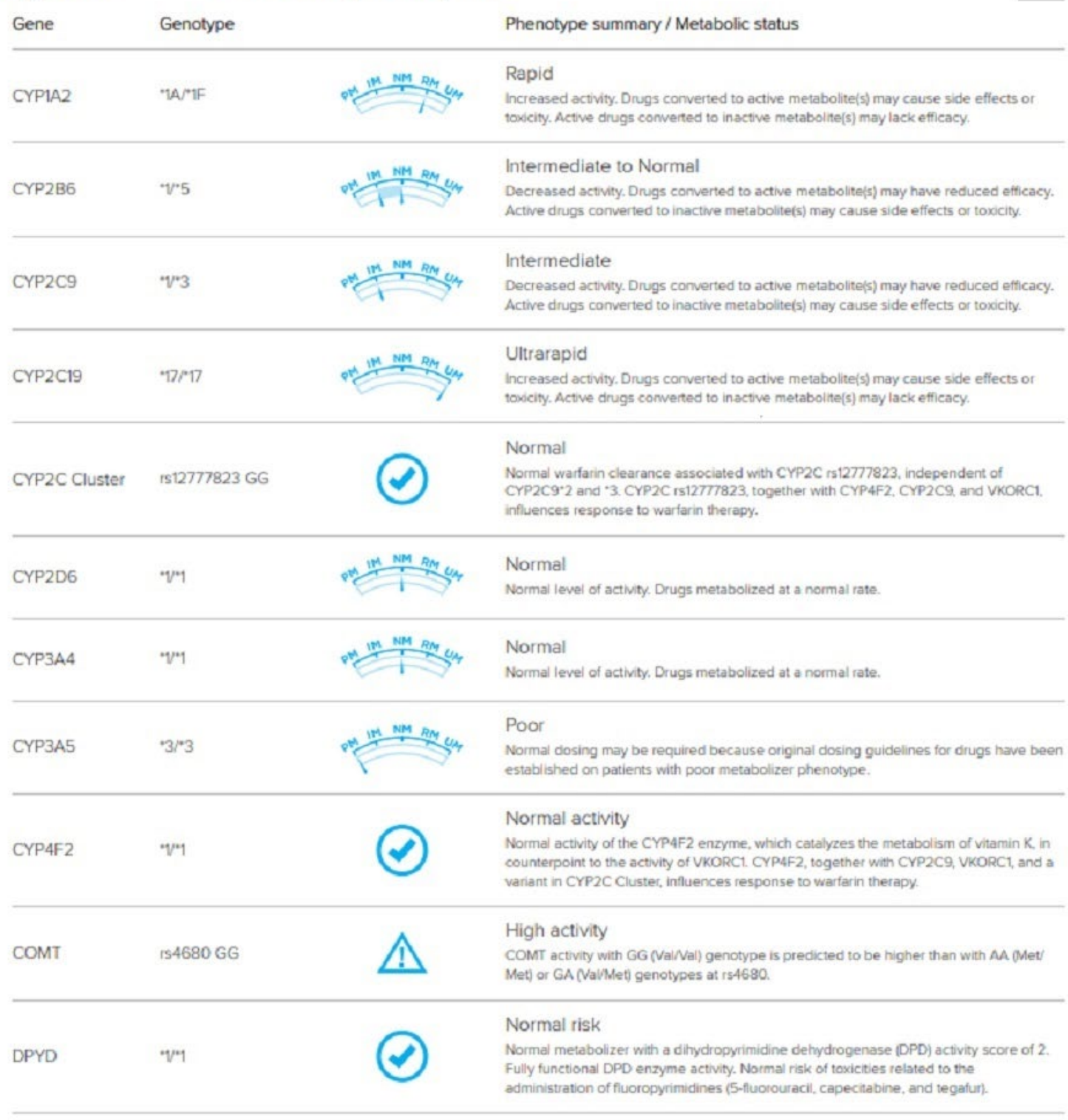

Source: www.OneOme.com ${ }^{14}$

http://z.umn.edu/INNOVATIONS

2019, Vol. 10, No. 3, Article 5

INNOVATIONS in pharmacy

DOI: https://doi.org/10.24926/iip.v10i3.2013

5 